\title{
Longitudinal Trend of Health-Related Quality of Life During Concurrent Chemoradiotherapy and Survival in Patients With Stage II-IVb Nasopharyngeal Carcinoma
}

\section{OPEN ACCESS}

Edited by:

Lorenzo Manti,

University of Naples Federico II, Italy

Reviewed by:

Maria Quarto,

University of Naples Federico II, Italy Vinay Sharma,

University of the Witwatersrand, South Africa

*Correspondence: Hai-Qiang Mai maihq@sysucc.org.cn

†These authors have contributed equally to this work

Specialty section: This article was submitted to

Radiation Oncology,

a section of the journal

Frontiers in Oncology

Received: 02 July 2020 Accepted: 08 September 2020 Published: 08 October 2020

Citation:

Li J-B, Guo S-S, Tang L-Q, Guo L, Mo $H-Y$, Chen $Q-Y$ and Mai $H-Q$ (2020) Longitudinal Trend of Health-Related Quality of Life During Concurrent Chemoradiotherapy and Survival in Patients With Stage II-IVb

Nasopharyngeal Carcinoma.

Front. Oncol. 10:579292.

doi: 10.3389/fonc.2020.579292

\begin{abstract}
Ji-Bin $\mathrm{Li}^{1,2+}$, Shan-Shan Guo ${ }^{2,3+}$, Lin-Quan Tang ${ }^{2,3}$, Ling Guo ${ }^{2,3}$, Hao-Yuan $\mathrm{Mo}^{2,3}$, Qiu-Yan Chen ${ }^{2,3}$ and Hai-Qiang Mai ${ }^{2,3 *}$
\end{abstract}

${ }^{1}$ Department of Clinical Research, Sun Yat-sen University Cancer Center, Guangzhou, China, ${ }^{2}$ State Key Laboratory of Oncology in South China, Guangdong Key Laboratory of Nasopharyngeal Carcinoma Diagnosis and Therapy, Collaborative Innovation Center for Cancer Medicine, Sun Yat-sen University Cancer Center, Guangzhou, China, ${ }^{3}$ Department of Nasopharyngeal Carcinoma, Sun Yat-sen University Cancer Center, Guangzhou, China

Background and Aims: To investigate the longitudinal trend of health-related quality of life (HRQOL) from the start to the end of concurrent chemoradiotherapy and survival in patients with advanced nasopharyngeal carcinoma (NPC).

Methods: A total of 145 patients with stage II-IVb NPC, who were a subsample of a randomized phase III clinical trial, were recruited in this study. HRQOL was measured weekly for a total of 6 weeks during concurrent chemoradiotherapy by the Chinese version of the European Organization for Research and Treatment of Cancer Quality of Life Questionnaire core 30. Longitudinal trends of HRQOL domains over time were analyzed using mixed models. Survival rates were estimated using Kaplan-Meier method.

Results: During a median follow-up of 45 months, the 3-year progression-free survival rate, overall survival rate, and distant metastasis-free survival rate were highly at $86.8 \%$ (95\% Cl: 80.1\%, 91.4\%), 95.1\% (95\% Cl: 90.1\%, 97.6\%), and 91.0\% (95\% Cl: 84.9\%, $94.6 \%)$, respectively. The average weekly declines of five functioning domains were 1.83 3.52 points during the treatment period, with role functioning having the largest decline rate $(-2.52$ points per week, 95\% Cl: $-4.50,-2.55 ; p<0.001)$. Loss of appetite is the most affected symptom, with severe appetite loss ranging from 35.9 to $61.1 \%$. The average increases of symptoms were $0.63-5.16$ points per week during treatment period (all $p$-values for time $<0.001$, except for financial difficulties), with pain symptoms having the largest increase $(5.16$ points, $95 \% \mathrm{Cl}: 4.25,6.08 ; p<0.001)$, followed by fatigue (3.62 points, 95\% Cl: 2.90, 4.35; $p<0.001$ ).

Conclusion: The HRQOL of patients with advanced NPC is poor and substantially deteriorated during the concurrent chemoradiotherapy (CCRT) period. Psychological care and support is necessary for patients with advanced NPC during the treatment period.

Keywords: longitudinal trend, health-related quality of life, cheomotherapy, nasopharyngeal carcinoma, radiotherapy 


\section{INTRODUCTION}

Nasopharyngeal carcinoma (NPC) is a malignant tumor arising from nasopharynx epithelium with an extremely unbalanced geographical global distribution. There were about 129,000 new cases worldwide in 2018, with more than $70 \%$ of new cases in East and Southeast Asia $(1,2)$. In China, the world agestandardized incidence rate of NPC was $2.17 / 100,000$, and the highest rate was observed in Southern China from Guangdong and Guangxi province $(3,4)$. Most patients with NPC were in stage II-IVb at initial diagnosis. Concurrent chemoradiotherapy (CCRT), recommended by the National Comprehensive Cancer Network, is the standard treatment for patients with stage II-IVb NPC (5). Some studies have indicated that the local control rate and 5-year overall survival rate of patients with NPC are up to 90 and $80 \%$, respectively $(6,7)$. However, the complications and treatment-related adverse effects are still non-ignorable. Patients with NPC are significantly affected by difficulties in swallowing, hearing loss, xerostomia, speech impediments, and psychological issues (e.g., depression, anxiety), which further aggravate their health-related quality of life (HRQOL) $(8,9)$.

The majority of previous studies mainly focused on the endpoints of overall survival, progression-free survival, or local control rate from the physician's point of view. In recent years, HRQOL has been recognized as an important treatment endpoint from patients' experience to perform a comprehensive evaluation and has been increasingly used in oncology trials for clinical decision making (10). HRQOL is an important outcome for patients with head and neck cancers, especially for NPC. The diagnosis and treatment of NPC is a life-threatening event, and patients with locally advanced NPC experience distressing issues such as pain, loss of appetite, and impairment in social and role functioning in terms of HRQOL at diagnosis (11). These problems are common not only at diagnosis and during treatment, but also for several years after (12). Pretreatment HRQOL has been reported as a predictor of survival for patients with NPC $(13,14)$ and other advanced cancers [e.g., lung cancer (15), breast cancer (16), colorectal cancer (17), and hepatocellular carcinoma (18)]. A change in scores of many HRQOL domains from initial to 6 months after radiation therapy has been significantly associated with overall survival in patients with head and neck cancers (19). Results has also shown that HRQOL (i.e., physical functioning, fatigue, appetite loss) after treatment significantly predicted disease-free survival and overall survival in patients with NPC (20). In addition, it was found that global quality of life, insomnia, and fatigue were significant predictors of weight loss (21), which has been associated with poor survival of patients with NPC $(22,23)$. Therefore, maintaining a high level of HRQOL during the treatment period is important for patient prognosis and psychological well-being. It has been reported that advanced NPC patients who received CCRT reported worse HRQOL compared to those who received radiotherapy (24). HRQOL in head and neck cancer patients deteriorates immediately after treatment and then gradually recovers to pretreatment levels at around 12 months after treatment (25). To the best of our knowledge, the variation trend of HRQOL during the CCRT period has not yet been established among patients with NPC. Such evidence may be helpful for physicians to act preventively or come up with recommendations for improving HRQOL in patients with NPC during and after the treatment period.

In this study, we explored the longitudinal trend of HRQOL during the CCRT period and survival among patients with II-IVb NPC, using the longitudinal data from a randomized phase III clinical trial (26). It is hypothesized that HRQOL would gradually deteriorate during the CCRT period.

\section{METHODS}

\section{Study Design and Participants}

Participants in this study were a subsample from a multicenter, open label, non-inferiority, randomized phase III trial (26). In the trial, between 16 January 2012 and 16 July 2014, a total of 402 patients aged 18-65 years with stage II-IVb NPC, a Karnofsky score of $\geq 70$, and adequate hematological, renal, and hepatic function were randomly assigned (1:1) to intravenously receive either nedaplatin $\left(100 \mathrm{mg} / \mathrm{m}^{2}\right)$ or cisplatin $\left(100 \mathrm{mg} / \mathrm{m}^{2}\right)$ on days 1,22 , and 43 for three cycles concurrently with intensity-modulated radiotherapy. The exclusion criteria included previous radiotherapy or chemotherapy for NPC; the presence of relapse or distant metastasis; a previous malignancy (apart from carcinoma in situ of the cervix, or basal or squamous cell carcinoma of the skin); the presence of uncontrolled lifethreatening illness; pregnancy or lactation; and any mental disorder or somatic comorbidities of clinical concern.

Among the 402 randomized patients, 145 (36\%) returned completed the European Organization for Research and Treatment of Cancer Quality of Life Questionnaire core 30 (EORCT QLQ-C30) surveys at baseline (before radiotherapy), making them suitable for the current study. The study was approved by the ethics committee or institutional review board at each participating center, and all patients provided written consent.

\section{Data Collection Procedures}

All patients were randomly assigned to receive intravenous nedaplatin or nedaplatin $\left(100 \mathrm{mg} / \mathrm{m}^{2}\right)$ on days 1,22 , and 43 for three cycles concurrently with intensity-modulated radiotherapy (2.00-2 33 Gy per fraction with five daily fractions per week for 6-7 weeks) (26).

HRQOL was assessed using the EORTC QLQ-C30, version 3.0 (27). Its Chinese version has been validated in a previous study (28). The EORTC QLQ-C30 is a 30-item generic cancer instrument which evaluates a global quality of life (QoL), five multi-item functioning scales (i.e., physical, role, emotional, cognitive, and social functioning), three multiitem symptom scales (fatigue, pain, and nausea/vomiting), and six single symptom items (dyspnea, insomnia, appetite loss, constipation, diarrhea, and financial difficulties). HRQOL scales were summarized as standard scores ranging from 0 to 100 according to the scoring manual (29). A higher score for global QoL and functioning scales represents a better level of global QoL or functioning, whereas a higher score for symptom scales/items indicates a higher level of symptomatology/problems. 
EORTC QLQ-C30 was self-administered weekly for a total of 6 weeks during the CCRT period. All assessments were carried out by a well-trained clinical research coordinator at the clinics. Sociodemographic characteristics were collected at the recruitment interview. After completion of treatment, participants were followed up at least every 3 months during the first 3 years and every 6 months thereafter until death. Progression-free survival was assessed by the investigator and defined as the time from the date of randomization to documented local or regional relapse, distant metastasis, or death from any cause, whichever occurred first. Overall survival was defined as the time from the date of randomization to death from any cause or censored at the date of last follow-up. Distant metastasis-free survival was defined as the time from the date of randomization to distant metastasis, or death from any cause. The censored date of the study was 31 June 2017.

\section{Statistical Analysis}

Descriptive analysis was presented as mean with standard deviation or frequency with percentage when appropriate. The longitudinal trend of the HRQOL scale scores from the beginning to the end of treatment was analyzed with mixed models using restricted maximum likelihood estimation and an unstructured covariance structure. Each mixed model included one of the HRQOL domains as a dependent variable, an intercept, and an independent variable representing time points during CCRT period, by univariable and adjustment of sociodemographic and clinical covariates separately. Two random effects were included in the mixed models: a random patient effect representing an individual baseline HRQOL (intercept) and a random subject by time effect respecting an individual linear change per week during the treatment period (slope of time variable). Regression coefficients along with $95 \%$ confidence interval (95\% CI) of time were reported.

Given that scores of $\leq 50$ for global QoL and functioning scales or scores of $>50$ for symptom scales/items indicate a need for intervention (30), we applied an absolute threshold value of 50 points for describing very low global QoL and functioning scores as worse global QoL / functioning or very high symptom scores as severe symptoms. The distributions of worse HRQOL were presented stratified by measurement time points.

All statistical analyses were performed using SAS for windows version 9.4 (SAS Institute, Cary, NC, USA). A two-sided $p<0.05$ was considered statistically significant. The original randomized phase III clinical trial is registered on ClinicalTrials.gov with number NCT01540136 (26). The key raw data of this study have been uploaded onto the Research Data Deposit platform (RDD), with approval number RDDA2018000932.

\section{RESULTS}

\section{Social-Demographic and Clinical Characteristics}

Among 145 patients at baseline, 51.7\% received cisplatin-based CCRT, and $48.3 \%$ received nedaplatin-based CCRT. The mean age of the patients was 44.3 years old (standard deviation: 9.8),
TABLE 1 | Sample characteristics.

\begin{tabular}{|c|c|c|}
\hline & $n$ & $\%$ \\
\hline Age at randomization, years, mean $\pm S D$ & \multicolumn{2}{|c|}{$44.3 \pm 9.8$} \\
\hline$\leq 45$ & 80 & 55.2 \\
\hline$>45$ & 65 & 44.8 \\
\hline \multicolumn{3}{|l|}{ Sex } \\
\hline Male & 108 & 74.5 \\
\hline Female & 37 & 25.5 \\
\hline \multicolumn{3}{|l|}{ Smoking habit } \\
\hline No & 92 & 63.4 \\
\hline Yes & 53 & 36.6 \\
\hline \multicolumn{3}{|l|}{ Drinking habit } \\
\hline No & 109 & 75.2 \\
\hline Yes & 36 & 24.8 \\
\hline $\mathrm{BMl}, \mathrm{kg} / \mathrm{m}^{2}$, mean $\pm \mathrm{SD}$ & \multicolumn{2}{|c|}{$23.0 \pm 2.9$} \\
\hline$<18.5$ & 6 & 4.1 \\
\hline $18.5-24.9$ & 104 & 71.7 \\
\hline 25.0-29.9 & 34 & 23.5 \\
\hline$\geq 30$ & 1 & 0.7 \\
\hline $\begin{array}{l}\text { Percentage of weight loss during CCRT } \\
\text { period, mean } \pm \text { SD }\end{array}$ & \multicolumn{2}{|c|}{$-6.7 \% \pm 6.6 \%$} \\
\hline No change or increase & 25 & 17.2 \\
\hline$<5 \%$ & 37 & 25.5 \\
\hline $5-10 \%$ & 41 & 28.3 \\
\hline$>10 \%$ & 42 & 29.0 \\
\hline \multicolumn{3}{|l|}{ T stage } \\
\hline $\mathrm{T} 1$ & 1 & 0.7 \\
\hline T2 & 27 & 18.6 \\
\hline T3 & 90 & 62.1 \\
\hline T4 & 27 & 18.6 \\
\hline \multicolumn{3}{|l|}{ N stage } \\
\hline NO & 14 & 9.7 \\
\hline N1 & 56 & 38.6 \\
\hline N2 & 66 & 45.5 \\
\hline N3 & 9 & 6.2 \\
\hline \multicolumn{3}{|l|}{ AJCC stage } \\
\hline ॥ & 15 & 10.5 \\
\hline III & 95 & 65.5 \\
\hline IV & 35 & 24.0 \\
\hline \multicolumn{3}{|l|}{ Epstein-Barr virus DNA test } \\
\hline DNA $<1,500$ copies per $\mathrm{mL}$ & 79 & 54.5 \\
\hline DNA $\geq 1,500$ copies per $\mathrm{mL}$ & 66 & 45.5 \\
\hline \multicolumn{3}{|l|}{ Intervention } \\
\hline Cisplatin & 75 & 51.7 \\
\hline Nedaplatin & 70 & 48.3 \\
\hline \multicolumn{3}{|l|}{ Chemotherapy cycles } \\
\hline Two or less & 39 & 26.9 \\
\hline Three & 106 & 73.1 \\
\hline \multicolumn{3}{|l|}{ Duration of radiotherapy } \\
\hline$\leq 42$ days & 55 & 37.9 \\
\hline$>42$ days & 90 & 62.1 \\
\hline
\end{tabular}

CCRT, concurrent chemoradiotherapy; AJCC, American joint committee on cancer; SD, standard deviation. 


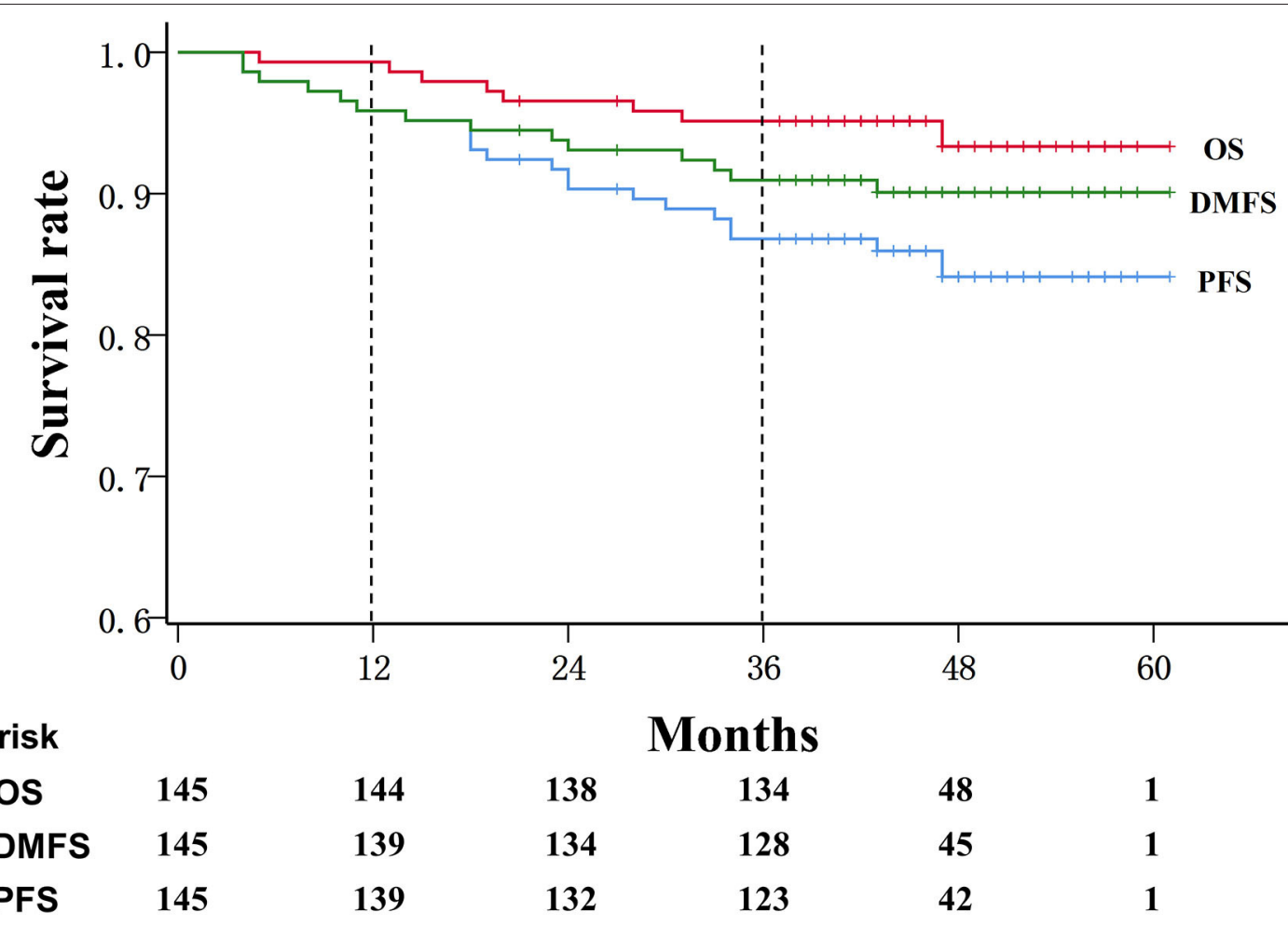

FIGURE 1 | Kaplan-Meier survival curve in patients with advanced nasopharyngeal carcinoma. OS, overall survival; DMFS, Distant metastasis-free survival; PFS, progression-free survival.

$74.5 \%$ of whom were male, $36.6 \%$ had a history of smoking, and $24.8 \%$ had a history of drinking. Around $24.2 \%$ of patients were overweight or obese at baseline, and more than half of patients $(57.3 \%)$ were observed to experience $5 \%$ or more of weight loss during the treatment period as compared to their baseline bodyweight (Table 1).

\section{Treatment Completion}

All of 145 patients completed the recommended radiotherapy. The median dose of RT was 70 Gy (Range: 70-70 Gy), and the median dose per fraction was 2.33 Gy (Range: 2.12$2.33 \mathrm{~Gy})$. Almost all patients $(99.3 \%, 144 / 145)$ received at least two cycles of chemotherapy, with $73.1 \% \quad(106 / 145)$ of patients completed the three cycles. Besides, $38.6 \%$ $(56 / 145)$ received chemotherapy with dosage $300 \mathrm{mg} / \mathrm{m}^{2}$, and $93.8 \%(136 / 145)$ patients received chemotherapy with dosage $\geq 200 \mathrm{mg} / \mathrm{m}^{2}$.

\section{Survival Rate}

During a median follow-up of 45 months, the progression-free survival rate was $95.9 \%$ (95\% CI: $91.0,97.7 \%$ ) at 1 year and $86.8 \%$ (95\% CI: 80.1, 91.4\%) at 3 years, and the overall survival rate was 99.3\% (95\% CI: 95.2, 99.9\%) at 1 year and 95.1\% (95\% CI: 90.1, $97.6 \%)$ at 3 years, whereas the distant metastasis-free survival rate was $95.9 \%$ (95\% CI: 91.0, 98.1\%) at 1 year and $91.0 \%$ (95\% CI: $84.9,94.6 \%$ ) at 3 years (Figure 1).

\section{HRQOL Completion}

Of 145 patients who completed baseline surveys, 96 (66.2\%), 139 (95.9\%), 106 (73.1\%), 128 (88.2\%), and 144 (99.3\%) provided valid data at week 2 to week 6 during the CCRT period, respectively. The attrition rate was the greatest at week 2 of the CCRT period. Participants were more likely to miss EORTC QLQ-C30 questionnaires at week 2 if they received two or less cycles of chemotherapy, were progression-free, were alive or were distant metastasis-free during the study period, while there were no significant differences of other sample characteristics between participants with and without EORTC QLQ-C30 questionnaires at week 2 (see online Supplementary Table 1). A total of $62.8 \%$ of patients completed all six-point assessments, $89.7 \%$ patients completed more than three assessments, and only $2.1 \%$ missed four of six assessments during the treatment period.

\section{Trend of Global QoL and Functioning Domains}

After adjusting the social-demographic and clinical characteristics listed in Table 1, the mixed models indicated a substantial deterioration across the 6-week treatment period in global QoL and five functioning domains. The global QoL had the lowest values compared to the five functioning domains in all six time points from 57.6 to 46.6 , with an average decline of 2.18 points per week (95\% CI: $-3.07,-1.30)$. (Table 2 and Figure 2A). 
TABLE 2 | Temporal trend of health-related quality of life (HRQOL) during the concurrent chemoradiotherapy period using the mixed model.

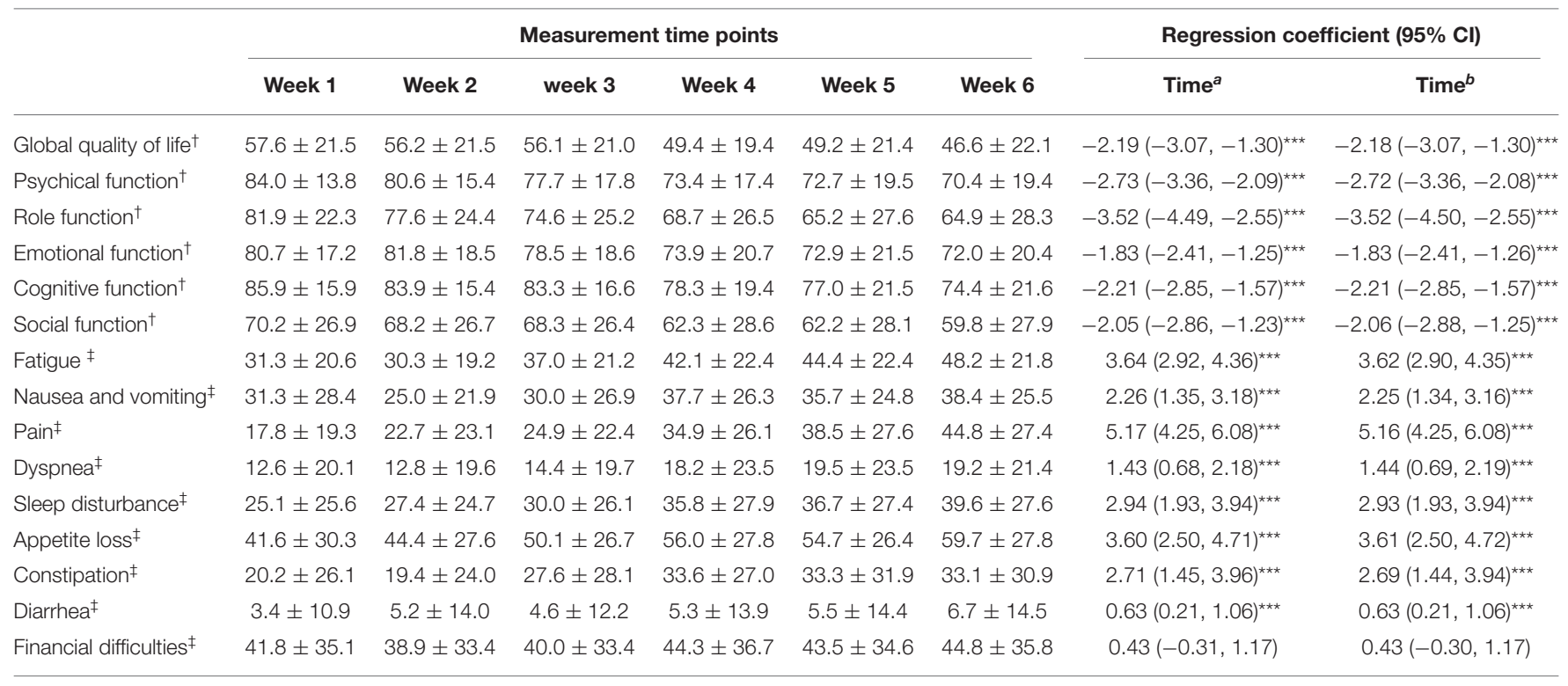

95\% Cl: 95\% confidence interval.

† Scale 0-100: higher score represents better quality of life.

¥ Scale 0-100: higher score represents more severe symptoms.

a regression coefficients obtained by univariable mixed models.

${ }^{b}$ regression coefficients obtained by mixed models after adjusting of age ( $\leq 45 \mathrm{vs.}>45$ ), sex (male vs. female), smoking habit (no vs. yes), drinking habit (no vs. yes), BMl ( $<25 \mathrm{~kg} / \mathrm{m}^{2}$ vs. $\geq 25 \mathrm{~kg} / \mathrm{m}^{2}$ ), weight loss during CCRT (no change/increase, <5\%, 5-10\%, >10\%), T stage (T1-2, T3, T4), N stage (NO-1 vs. N2-3), AJCC stage (II, III, IV), pretreatment Epstein-Barr virus DNA copies ( $<1,500$ vs. $\geq 1,500$ ), intervention arm (cisplatin vs. nedaplatin), number of chemotherapy cycles ( $\leq 2$ cycles vs. $>2$ cycles), and duration of radiotherapy ( $\leq 42$ days vs. $>42$ days).

${ }^{* * *} p<0.001$.

The average declines per week of the five functioning domains were 1.83-3.52 points across the CCRT period. Role functioning had the largest decline rate $(-2.52$ points per week, $95 \%$ CI: $-4.50,-2.55 ; p<0.001)$, followed by physical functioning $(-2.72$ points per week, 95\% CI: $-3.36,-2.08 ; p<0.001)$. Cognitive functioning remained at a relatively higher level during the treatment period. Social functioning and role functioning had the lowest values compared to the other three functioning domains. (Table 2 and Figure 2A).

The proportion of patients who scored $\leq 50$ points in global QoL had a relatively high level at all six time points, increasing significantly from $42.8 \%$ at week 1 to $64.6 \%$ at week 6 . The proportion of worse functioning (scores $\leq 50$ points) increased from 1.4 to $15.3 \%$ for physical functioning, from 15.3 to $33.3 \%$ for role functioning, from 7.6 to $16.8 \%$ for emotional functioning, from 4.1 to $15.4 \%$ for cognitive functioning, and from 24.8 to $35.2 \%$ for social functioning during the treatment period (Figures 3A-F).

\section{Trend of Symptoms}

The domain of financial difficulties remained at a stable and medium level from 41.8 points at week 1 to 44.8 points at the end of treatment, whereas the other eight symptoms significantly increased during the treatment period. The average increase of symptoms was $0.63-5.16$ points per week during the treatment period (all $p$-values for time $<0.001$ ), with pain symptoms having the largest increase (5.16 points, $95 \%$ CI: $4.25,6.08$; $p<$
0.001), followed by fatigue (3.62 points, $95 \%$ CI: $2.90,4.35$; $p<$ 0.001 ). The diarrhea symptom had the lowest values in the nine symptom domains during the treatment period, with a slight but significant increase from 3.4 to $6.7(p<0.001)$. The first three prominent symptoms were loss of appetite, financial difficulties, and fatigue (Table 2 and Figure 2B).

The proportion of patients with severe diarrhea symptoms (from 0.7 to $1.4 \%$ ) and dyspnea (from 3.4 to $6.9 \%$ ) were small during the treatment period compared to those of the other symptoms. The proportion of patients with severe loss of appetite (from 35.9 to $61.1 \%$ ), fatigue (from 13.8 to $43.8 \%$ ), pain (from 2.8 to $32.9 \%$ ), and sleep disturbance (from 14.5 to $34.0 \%$ ) largely increased during the treatment period. (Figure 3G-O).

\section{DISCUSSION}

In this longitudinal study, the survival rate of patients with advanced NPC is very high, which is similar to previous studies $(31,32)$. We observed a substantially deteriorated trend among all domains except financial difficulties in terms of HRQOL during the CCRT period. Global QoL largely declined, with more than $64 \%$ of patients scoring a severely worse level at the end of CCRT. This indicates that CCRT significantly degrades HRQOL in patients with advanced NPC. As a radiosensitive cancer, radiation-related toxicity and complications (e.g., neuropathy, hearing loss, and xerostomia) could reduce HRQOL in patients with NPC $(8,33)$. Previous studies have outlined a deterioration 

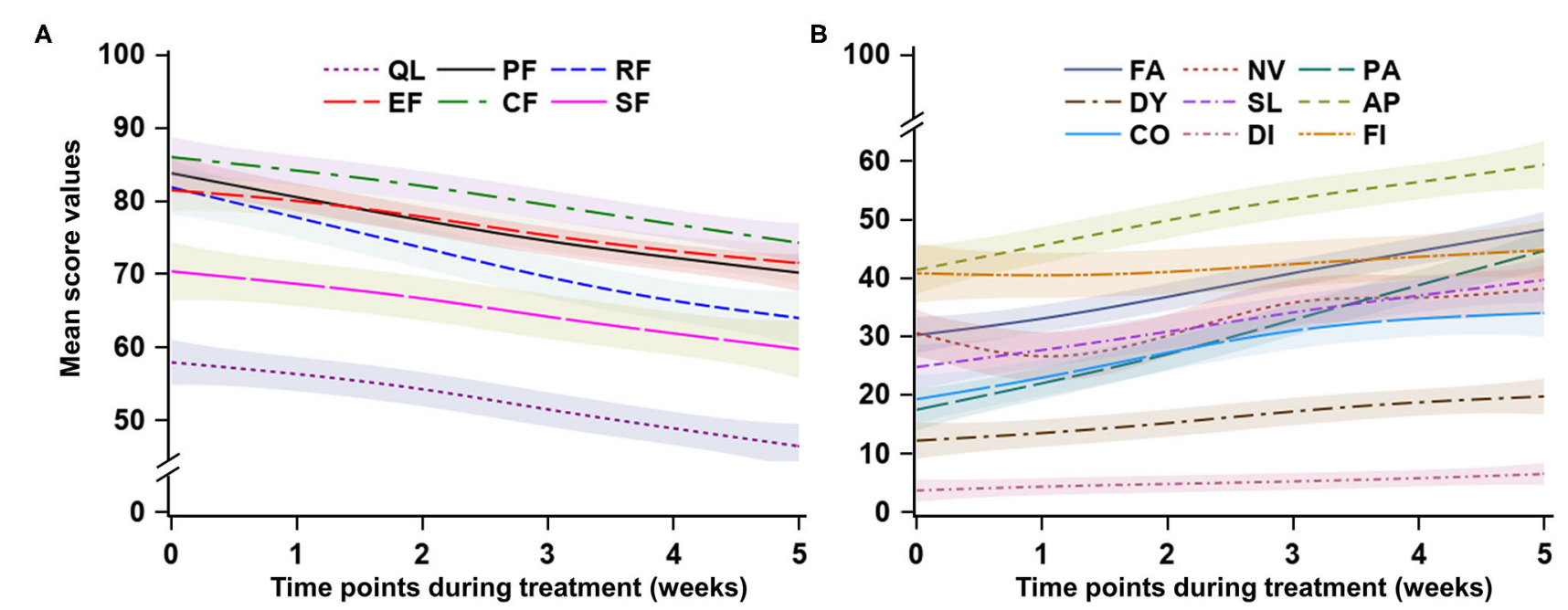

FIGURE 2 | Trend of health-related quality of life during the concurrent chemoradiotherapy period. QL, global quality of life; PF, physical functioning; RF, role functioning; EF, emotional functioning; CF, cognitive functioning; SF, social functioning; FA, fatigue; NV, nausea and vomiting; PA, pain; DY, dyspnea; SL, sleep disturbance; AP, appetite loss; CO, constipation; DI, Diarrhea; FI, financial difficulties. For (A), a higher score represents better quality of life or functioning; for (B), a higher score represents more severe symptoms.

of HRQOL during the first 3 months after the initial treatment in patients with head and neck cancers $(19,34)$. Therefore, more psychological care and support is necessary for patients with advanced NPC during the CCRT period. Physicians should take necessary actions to improve the HRQOL of patients with NPC during the CCRT period.

We found that social functioning and role functioning are two of the most affected functioning domains by NPC and chemoradiotherapy, with role functioning having the largest decline in the five functioning domains. The findings were consolidated by the report of Hammerlid et al. reporting that patients with NPC had the worst social and role functioning compared to those with other head and neck cancers (11). Similarly, a previous study reported that role emotional and social function, which were measured by the Shot Form 36 Health Survey Questionnaire, were lower at week 3 of radiotherapy than those before therapy (35). Social functioning was also reported as the lowest of the five functioning domains among patients with recurrent NPC (36). This might be explained by the effects of symptoms and complaints (e.g., cancer-related pain, fatigue, xerostomia) caused by radiotherapy and chemotherapy. NPC patients with severe symptoms and side effects might avoid social eating and interactions with friends/relatives, and then be vulnerable to social difficulties and interpersonal and role maladjustment.

The symptom of pain is common among head and neck cancer patients. A pooled prevalence of pain is over $50 \%$ in all cancer types, with the highest prevalence of $70 \%$ in head and neck cancer patients (37). Similarly, in our study, pain is the largest worsening symptom in NPC patients during the CCRT period, with the percentage of those experiencing severe pain sharply increasing from $2.8 \%$ at the beginning to $32.9 \%$ at the end of CCRT. Oral mucositis, which was highly prevalent among
NPC patients who received radiotherapy, might be the major source of pain (38). Pain could substantially and adversely affect patients' quality of life, adherence to therapy, treatment efficacy, and satisfaction with care, and might be a clinically indicator of tumor progression $(39,40)$. Therefore, pain management should be vital and considered in cancer care, which could substantially improve the patient-perceived value of cancer treatment (40).

We found that loss of appetite and fatigue are two of the most severe symptoms during the CCRT treatment. Mouth dryness, tasted changes, salivary gland damage, pain, and difficulties in swallowing are common adverse effects caused by radiotherapy, which undoubtedly induce patients' loss of appetite and eating difficulties (41) and then further caused malnutrition (42). Cancer-related fatigue is significantly associated with patients' psychological distress and poor quality of life, and is a risk factor for reduced survival (43). In our study, fatigue largely deteriorated during the CCRT period. It was reported that 30 to $60 \%$ of cancer patients suffer from moderate to severe fatigue during the treatment, which may reduce treatment efficacy due to non-compliance with treatment (44).

Currently, cancer-related symptoms (i.e., pain, loss of appetite, fatigue) are still undertreated and poorly controlled in clinical practices $(40,45)$. The findings in our study can provide useful information for physicians conducting CCRT in patients with advanced NPC. Although the benefit of better survival for advanced NPC through CCRT has been well-confirmed in our study and previous reports, it is necessary to provide appropriate support and management for such patients to improve their HRQOL and psychological well-being during the CCRT period. These findings indicate that advanced NPC patients suffered from severe symptoms (i.e., appetite loss, fatigue, and pain) and the associated functional limitations. The deteriorating trend of HRQOL during the CCRT period could serve as an alert 


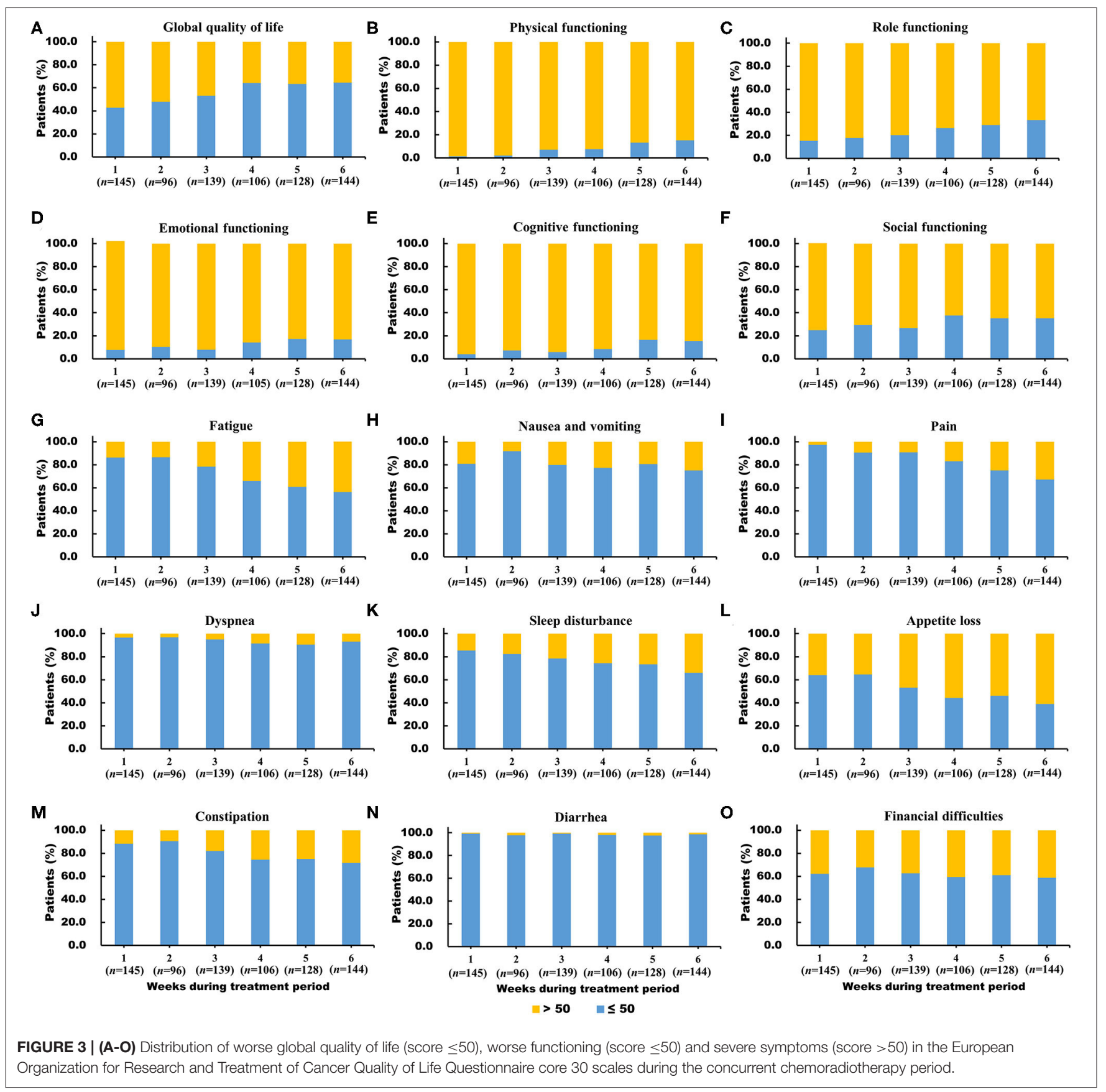

for physicians to provide effective and supportive care or refer patients to the appropriate services when necessary.

The main strength of our study is that HRQOL was measured weekly with 6 time points from the beginning to the end of treatment, which allowed us to explore the longitudinal trend and the changes in HRQOL during the CCRT period more precisely and robustly. Another major advantage is that the design and homogeneity of patients along with data quality are robust, as our study is based on a randomized phase III clinical trial (26). However, there are some limitations when interpreting the results in the present study. First, the sample size in this study is relatively small, and the findings is warranted for further confirmation by large-sample prospective studies. Second, due to the short-term period of follow-up in this study, only a small number of events were observed during the study period (i.e., death, disease progression, and distant metastasis), which did not allow us to estimate the potential effects of impaired HRQOL during the treatment period on subsequent survival outcomes among patients with advanced NPC. Third, it is necessary to highlight that our findings could only reflect the trend of HRQOL during the treatment period. Considering that HRQOL is highly dynamic during and 
after treatment, the longitudinal trend after treatment is still unclear. Fourth, there may have selection bias considering the large attrition rate. The large attrition rate might be partially attributable to the better treatment effect and lower treatmentrelated toxicities during the CCRT period, as these patients may feel well and did not present in the clinics for follow-up as scheduled. Hence, the assumption that missing data are missing at random might not be appropriate in this study (29). HRQOL during CCRT period among patients with advanced NPC might be underestimated.

In conclusion, our study revealed that HRQOL in patients with advanced NPC is poor and largely deteriorated during the CCRT period. Social functioning and role functioning are two of the most affected functioning domains, while loss of appetite, fatigue, and pain are the three major symptoms during the CCRT period. These findings are useful for clinicians in conducting relevant clinical treatment and in designing interventions for future studies. Longitudinal studies that measure HRQOL during and after treatment over a long time frame are highly warranted to explore the long-term trends of HRQOL and their impact on survival outcomes among patients with NPC.

\section{DATA AVAILABILITY STATEMENT}

The raw data supporting the conclusions of this article will be made available by the authors, without undue reservation.

\section{ETHICS STATEMENT}

The studies involving human participants were reviewed and approved by the ethics committee of Sun Yetsan University Cancer Center. The patients/participants provided their written informed consent to participate in this study.

\section{REFERENCES}

1. Bray F, Ferlays J, Soerjomataram I, Siegel RL, Torre LA, Jemal A. Global cancer statistics 2018: GLOBOCAN estimates of incidence and mortality worldwide for 36 cancers in 185 countries. CA Cancer J Clin. (2018) 68:394424. doi: $10.3322 /$ caac. 21492

2. Chen YP, Chan ATC, Le QT, Blanchard P, Sun Y, Ma J. Nasopharyngeal carcinoma. Lancet. (2019) 394:64-80. doi: 10.1016/S0140-6736(19)30956-0

3. Wei KR, Zheng RS, Zhang SW, Liang ZH, Li ZM, Chen WQ. Nasopharyngeal carcinoma incidence and mortality in China, 2013. Chinese J Cancer. (2017) 36:90. doi: 10.1186/s40880-017-0257-9

4. Cao SM, Simons MJ, Qian CN. The prevalence and prevention of nasopharyngeal carcinoma in China. Chin J Cancer. (2011) 30:1149. doi: $10.5732 /$ cjc. 010.10377

5. Network NCC. NCCN Guidelines: Head and Neck Cancers Version 2. NCCN (2020).

6. Zhang MX, Li J, Shen GP, Zou X, Xu JJ, Jiang R, et al. Intensity-modulated radiotherapy prolongs the survival of patients with nasopharyngeal carcinoma compared with conventional two-dimensional radiotherapy: a 10-year experience with a large cohort and long follow-up. Eur J Cancer. (2015) 51:2587-95. doi: 10.1016/j.ejca.2015.08.006

7. Lin S, Lu JJ, Han L, Chen Q, Pan J. Sequential chemotherapy and intensitymodulated radiation therapy in the management of locoregionally advanced

\section{AUTHOR CONTRIBUTIONS}

J-BL, H-QM, and S-SG: study concepts. J-BL and H-QM: study design. J-BL, S-SG, and L-QT: data acquisition. S-SG, L-QT, LG, H-YM, and Q-YC: quality control of data and algorithms. J-BL: data analysis and interpretation and statistical analysis. J-BL and S-SG: manuscript preparation. J-BL, S-SG, and HQM: manuscript editing. H-QM: manuscript review. All authors contributed to the article and approved the submitted version.

\section{FUNDING}

This study was partly supported by grants from National Natural Science Foundation of China (Grant No. 81803105, 81425018,81672868 , and 81802775), Natural Science Foundation of Guangdong Province (Grant No. 2018A030310238 and 2017A030312003), Medical Science and Technology Research Fund of Guangdong Province (Grant No. A2018201), and the Sun Yat-sen University Clinical Research 5010 Program. The funding sources had no roles in the design and conduct of the study collection, management, analysis, and interpretation of the data; preparation, review, or approval of the manuscript; and decision to submit the manuscript for publication.

\section{ACKNOWLEDGMENTS}

We thank the patients who participated in this study and their families, as well as the medical, nursing, and research staff at the study centers.

\section{SUPPLEMENTARY MATERIAL}

The Supplementary Material for this article can be found online at: https://www.frontiersin.org/articles/10.3389/fonc. 2020.579292/full\#supplementary-material

nasopharyngeal carcinoma: experience of 370 consecutive cases. BMC Cancer. (2010) 10:39. doi: 10.1186/1471-2407-10-39

8. Tsai WL, Huang TL, Liao KC, Chuang HC, Lin YT, Lee TF, et al. Impact of late toxicities on quality of life for survivors of nasopharyngeal carcinoma. BMC Cancer. (2014) 14:856. doi: 10.1186/1471-2407-14-856

9. McDowell LJ, Rock K, Xu W, Chan B, Waldron J, Lu L, et al. Long-Term late toxicity, quality of life, and emotional distress in patients with nasopharyngeal carcinoma treated with intensity modulated radiation therapy. Int J Radiat Oncol Biol Phys. (2018) 102:340-52. doi: 10.1016/j.ijrobp.2018.05.060

10. Calvert M, Blazeby J, Revicki D, Moher D, Brundage M. Reporting quality of life in clinical trials: a CONSORT extension. Lancet. (2011) 378:16845. doi: 10.1016/S0140-6736(11)61256-7

11. Hammerlid E, Bjordal K, Ahlner-Elmqvist M, Boysen M, Evensen JF, Biorklund A, et al. A prospective study of quality of life in head and neck cancer patients. Part I: at diagnosis. Laryngoscope. (2001) 111 (4 Pt. 1):66980. doi: 10.1097/00005537-200104000-00021

12. Bjordal K, Kaasa S, Mastekaasa A. Quality of life in patients treated for head and neck cancer: a follow-up study 7 to 11 years after radiotherapy. Int J Radiat Oncol Biol Phys. (1994) 28:847-56. doi: 10.1016/0360-3016(94)90104-X

13. Guo SS, Hu W, Chen QY, Li JM, Zhu SH, He Y, et al. Pretreatment quality of life as a predictor of survival for patients with nasopharyngeal carcinoma treated with IMRT. BMC Cancer. (2018) 18:114. doi: 10.1186/s12885-018-4003-8 
14. Fang FM, Tsai WL, Chien CY, Chen HC, Hsu HC, Huang TL, et al. Pretreatment quality of life as a predictor of distant metastasis and survival for patients with nasopharyngeal carcinoma. J Clin Oncol. (2010) 28:43849. doi: 10.1200/JCO.2010.28.8324

15. Fiteni F, Vernerey D, Bonnetain F, Vaylet F, Sennélart H, Trédaniel J, et al. Prognostic value of health-related quality of life for overall survival in elderly non-small-cell lung cancer patients. Eur J Cancer. (2016) 52:1208. doi: 10.1016/j.ejca.2015.10.004

16. Efficace F, Therasse P, Piccart MJ, Coens C, van Steen K, Welnicka-Jaskiewicz $\mathrm{M}$, et al. Health-related quality of life parameters as prognostic factors in a nonmetastatic breast cancer population: an international multicenter study. $J$ Clin Oncol. (2004) 22:3381-8. doi: 10.1200/JCO.2004.02.060

17. Fournier E, Jooste V, Woronoff AS, Quipourt V, Bouvier AM, Mercier M. Health-related quality of life is a prognostic factor for survival in older patients after colorectal cancer diagnosis: a population-based study. Digest Liver Dis. (2016) 48:87-93. doi: 10.1016/j.dld.2015.09.006

18. Steel JL, Geller DA, Robinson TL, Savkova AY, Brower DS, Marsh JW, et al. Health-related quality of life as a prognostic factor in patients with advanced cancer. Cancer. (2014) 120:3717-21. doi: 10.1002/cncr.28902

19. Meyer F, Fortin A, Gélinas M, Nabid A, Brochet F, Têtu B, et al. Healthrelated quality of life as a survival predictor for patients with localized head and neck cancer treated with radiation therapy. J Clin Oncol. (2009) 27:2970-6. doi: 10.1200/JCO.2008.20.0295

20. Tsai WL, Chien CY, Huang HY, Liao KC, Fang FM. Prognostic value of quality of life measured after treatment on subsequent survival in patients with nasopharyngeal carcinoma. Qual Life Res. (2013) 22:71523. doi: 10.1007/s11136-012-0213-8

21. Qiu C, Yang N, Tian G, Liu H. Weight loss during radiotherapy for nasopharyngeal carcinoma: a prospective study from Northern China. Nutr Cancer. (2011) 63:873-9. doi: 10.1080/01635581.2011.582223

22. Zeng Q, Shen LJ, Guo X, Guo XM, Qian CN, Wu PH. Critical weight loss predicts poor prognosis in nasopharyngeal carcinoma. BMC Cancer. (2016) 16:169. doi: 10.1186/s12885-016-2214-4

23. Du XJ, Tang LL, Mao YP, Guo R, Sun Y, Lin AH, et al. Value of the prognostic nutritional index and weight loss in predicting metastasis and long-term mortality in nasopharyngeal carcinoma. J Transl Med. (2015) 13:364. doi: 10.1186/s12967-015-0729-0

24. Pan XB, Huang ST, Chen KH, Jiang YM, Ma JL, Qu S, et al. Concurrent chemoradiotherapy degrades the quality of life of patients with stage II nasopharyngeal carcinoma as compared to radiotherapy. Oncotarget. (2017) 8:14029-38. doi: 10.18632/oncotarget.14932

25. Høxbroe Michaelsen S, Grønhøj C, Høxbroe Michaelsen J, Friborg J, von Buchwald C. Quality of life in survivors of oropharyngeal cancer: a systematic review and meta-analysis of 1366 patients. Eur J Cancer. (2017) 78:91102. doi: 10.1016/j.ejca.2017.03.006

26. Tang LQ, Chen DP, Guo L, Mo HY, Huang Y, Guo SS, et al. Concurrent chemoradiotherapy with nedaplatin versus cisplatin in stage II-IVB nasopharyngeal carcinoma: an open-label, non-inferiority, randomised phase 3 trial. Lancet Oncol. (2018) 19:461-73. doi: 10.1016/S1470-2045(18)30104-9

27. Aaronson NK, Ahmedzai S, Bergman B, Bullinger M, Cull A, Duez NJ, et al. The European organization for research and treatment of cancer QLQ-C30: a quality-of-life instrument for use in international clinical trials in oncology. $J$ Natl Cancer Inst. (1993) 85:365-76. doi: 10.1093/jnci/85.5.365

28. Zhao H, Kanda K. Testing psychometric properties of the standard Chinese version of the European organization for research and treatment of cancer quality of life core questionnaire 30 (EORTC QLQ-C30). J Epidemiol. (2004) 14:193-203. doi: 10.2188/jea.14.193

29. Fayers PM, Aaronson N, Bjordal K, Groenvold M, Curran D, Bottomley A. On Behalf of the EORTC Quality of Life Group. The EORTC QLQ-C30 Scoring Manual. 3rd ed. Brussels: European Organisation for Research and Treatment of Cancer (2001).

30. Klinkhammer-Schalke M, Koller M, Ehret C, Steinger B, Ernst B, Wyatt JC, et al. Implementing a system of quality-of-life diagnosis and therapy for breast cancer patients: results of an exploratory trial as a prerequisite for a subsequent RCT. Br J Cancer. (2008) 99:415-22. doi: 10.1038/sj.bjc.6604505

31. You R, Sun R, Hua YJ, Li CF, Li JB, Zou X, et al. Cetuximab or nimotuzumab plus intensity-modulated radiotherapy versus cisplatin plus intensity-modulated radiotherapy for stage II-IVb nasopharyngeal carcinoma. Int J Cancer. (2017) 141:1265-76. doi: 10.1002/ijc.30819

32. You R, Hua YJ, Liu YP, Yang Q, Zhang YN, Li JB, et al. Concurrent chemoradiotherapy with or without anti-EGFR-targeted treatment for stage II-IVb nasopharyngeal carcinoma: retrospective analysis with a large cohort and long follow-up. Theranostics. (2017) 7:2314-24. doi: 10.7150/thno. 19710

33. Langendijk JA, Doornaert P, Verdonck-de Leeuw IM, Leemans CR, Aaronson NK, Slotman BJ. Impact of late treatment-related toxicity on quality of life among patients with head and neck cancer treated with radiotherapy. J Clin Oncol. (2008) 26:3770-6. doi: 10.1200/JCO.2007.14. 6647

34. Bjordal K, Ahlner-Elmqvist M, Hammerlid E, Boysen M, Evensen JF, Biörklund A, et al. A prospective study of quality of life in head and neck cancer patients. Part II: longitudinal data. Laryngoscope. (2001) 111:144052. doi: 10.1097/00005537-200108000-00022

35. Chen J, Liu P, Wang Q, Wu L, Zhang X. Influence of intensitymodulated radiation therapy on the life quality of patients with nasopharyngeal carcinoma. Cell Biochem Biophys. (2015) 73:731-6. doi: 10.1007/s12013-015-0638-0

36. Chan YW, Chow VL, Wei WI. Quality of life of patients after salvage nasopharyngectomy for recurrent nasopharyngeal carcinoma. Cancer. (2012) 118:3710-8. doi: 10.1002/cncr.26719

37. van den Beuken-van Everdingen MH, de Rijke JM, Kessels AG, Schouten HC, van Kleef M, Patijn J. Prevalence of pain in patients with cancer: a systematic review of the past 40 years. Ann Oncol. (2007) 18:143749. doi: $10.1093 /$ annonc/mdm056

38. Hua X, Chen LM, Zhu Q, Hu W, Lin C, Long ZQ, et al. Efficacy of controlled-release oxycodone for reducing pain due to oral mucositis in nasopharyngeal carcinoma patients treated with concurrent chemoradiotherapy: a prospective clinical trial. Support Care Cancer. (2019) 27:3759-67. doi: 10.1007/s00520-019-4643-5

39. Reis-Pina P, Lawlor PG, Barbosa A. Cancer-related pain management and the optimal use of opioids. Acta Medica Portuguesa. (2015) 28:37681. doi: 10.20344/amp.5801

40. Neufeld NJ, Elnahal SM, Alvarez RH. Cancer pain: a review of epidemiology, clinical quality and value impact. Future Oncol. (2017) 13:833-41. doi: 10.2217/fon-2016-0423

41. Fang FM, Chiu HC, Kuo WR, Wang CJ, Leung SW, Chen HC, et al. Healthrelated quality of life for nasopharyngeal carcinoma patients with cancerfree survival after treatment. Int J Radiat Oncol Biol Phys. (2002) 53:95968. doi: 10.1016/S0360-3016(02)02838-9

42. Jin T, Li KX, Li PJ, Huang S, Chen XZ, Chen M, et al. An evaluation of nutrition intervention during radiation therapy in patients with locoregionally advanced nasopharyngeal carcinoma. Oncotarget. (2017) 8:83723-33. doi: 10.18632/oncotarget.19381

43. Hofman M, Ryan JL, Figueroa-Moseley CD, Jean-Pierre P, Morrow GR. Cancer-related fatigue: the scale of the problem. Oncologist. (2007) 12 (Suppl. 1):4-10. doi: 10.1634/theoncologist.12-S1-4

44. Bower JE. Cancer-related fatigue-mechanisms, risk factors, and treatments. Nat Rev Clin Oncol. (2014) 11:597-609. doi: 10.1038/nrclinonc.2014.127

45. Vogelzang NJ, Breitbart W, Cella D, Curt GA, Groopman JE, Horning SJ, et al. Patient, caregiver, and oncologist perceptions of cancer-related fatigue: results of a tripart assessment survey. The fatigue coalition. Semin Hematol. (1997) 34 (3 Suppl. 2):4-12.

Conflict of Interest: The authors declare that the research was conducted in the absence of any commercial or financial relationships that could be construed as a potential conflict of interest.

Copyright $\odot 2020 \mathrm{Li}$, Guo, Tang, Guo, Mo, Chen and Mai. This is an open-access article distributed under the terms of the Creative Commons Attribution License (CC $B Y)$. The use, distribution or reproduction in other forums is permitted, provided the original author(s) and the copyright owner(s) are credited and that the original publication in this journal is cited, in accordance with accepted academic practice. No use, distribution or reproduction is permitted which does not comply with these terms. 